\title{
SOFT TISSUE INJURY IN EXTREME ROCK CLIMBERS
}

\section{S. R. BOLLEN}

ARC Boots Research Fellow, Rheumatism Research Unit, University of Leeds

\section{ABSTRACT}

Rock climbing is an increasingly popular sport. Its standards of difficulty have undergone a revolution in the past ten years. $\underset{乛}{\lessgtr}$ Regular training is now almost mandatory for the aspiring climber, but little has been published about the patterns of soft tissue injury to which climbers are susceptible. This paper aims to identify some of the common injuries that may be encountered, some of which do not appear to be associated with other sports.

\section{INTRODUCTION}

Rock climbing is an increasingly popular sport in this country and on a mild weekend, climbing "Meccas", such as Stannage Edge in Derbyshire or Llanberis pass in North Wales, can be seen to be swarming with its colourful devotees. The British Mountaineering Council now has 236 member clubs, 237 associate organisations and 4,358 individual paid-up members.

The last ten or fifteen years has seen an explosion in the sport's standards, and even the appearance of professional rock climbers and climbing competitions with large financial prizes. Hard modern climbing puts enormous strains on the upper limb (Fig. 1) and as some form of training has almost become mandatory, overuse injuries have become increasingly common. Many top climbers are training every day in the winter and climbing every possible day in the summer. Top rock climbing now requires the same sort of discipline as gymnastics, but has the added psychological pressure of being performed further from the ground.

Descriptions of the various fractures sustained during a serious fall may be found in any standard orthopaedic textbook, but thankfully this sort of serious injury has become rarer, as ropes, harnesses and protection equipment have become more sophisticated and improved in quality. At the present time however, no work could be found documenting the pattern of soft tissue injuries affecting the modern climber, some of which have not been described in association with other sports.

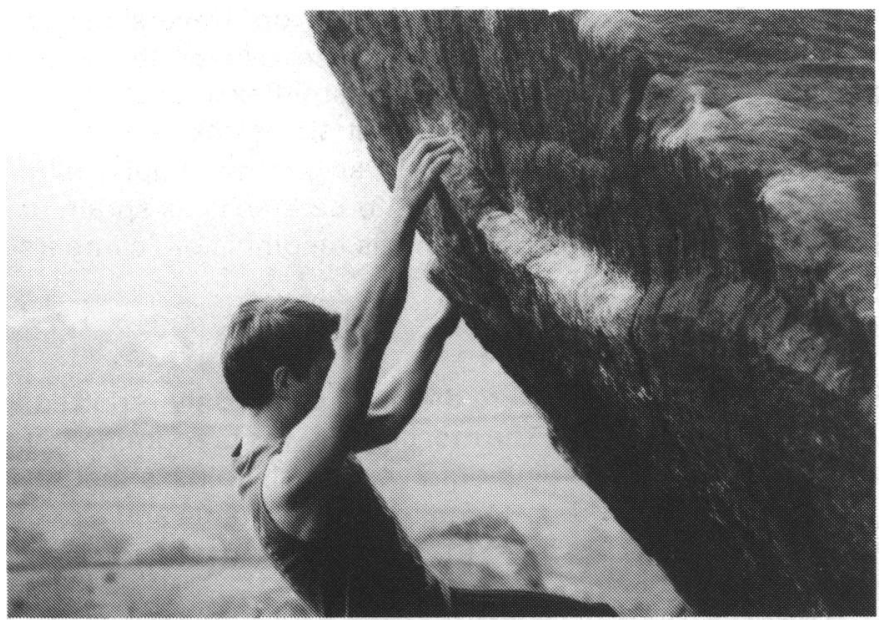

Fig. 1: A typical example of an overhanging pitch commonly tackled in modern rock climbing.

Address for correspondence:

Mr. S. R. Bollen

Rheumatism Research Unit

36 Clarendon Road

Leeds

LS2 9PJ

\section{MATERIALS AND METHODS}

A committee was set up by the British Mountaineering Council to investigate training methods in rock climbing. My brief was to look at the injuries sustained during intensive periods of climbing or training, so that by identifying the problem areas, appropriate training schedules could be devised.

Up to now, no guidelines have been available for the aspiring extreme climber and it would appear that overuse and training injuries have been fairly common. A questionnaire with diagrams (Fig. 2 and Appendix), was devised and circulated at a "Rock Symposium" at the national centre for outdoor activities at Plas-Y-Brenin. Additional questionnaires were circulated during subsequent training workshops, and the results analysed. This was supplemented by interviews with as many of the responders as possible. The questionnaire was restricted to climbers climbing at "extreme" standard (at E1 the footholds and handholds are about the size of the edge of a cassette box, at E6 they are about the size of the width of the tape), as it was felt that injury problems, and certainly training problems, would be more common in this group.

Out of a total of 100 questionnaires printed and distributed 86 were returned and analysed. Where no professional opinion had been sought, and diagnosis given, a tentative diagnosis was arrived at by analysing the mechanism and site of injury and the severity and duration of the pain.

\section{RESULTS}

There were 76 male and 10 female responders with a mean age of 27 (range 15-46), and a total of 115 injuries, symptomatic for ten days or longer, reported (Table I). Interestingly nearly half of these (54 as against 61) had occurred during training. The difficulty at which they were

TABLE I

Injuries by region

\begin{tabular}{lr}
\hline Lower Limb & 12 \\
Upper Limb & \\
Shoulder & 16 \\
Elbow & 6 \\
Med. epicondyle & 4 \\
Lat. epicondyle & 10 \\
Anterior & \\
Hand & 31 \\
Proximal interphalangeal joint & 12 \\
1st metacarpophalangeal joint & 9 \\
Wrist & 15 \\
Miscellaneous & 115 \\
Total & \\
\hline
\end{tabular}




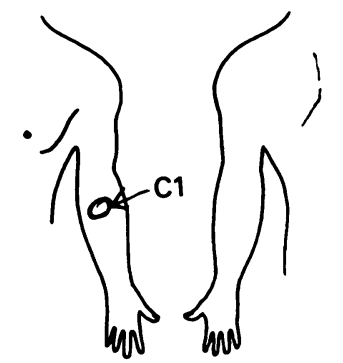

FRONT
LEFT
BACK
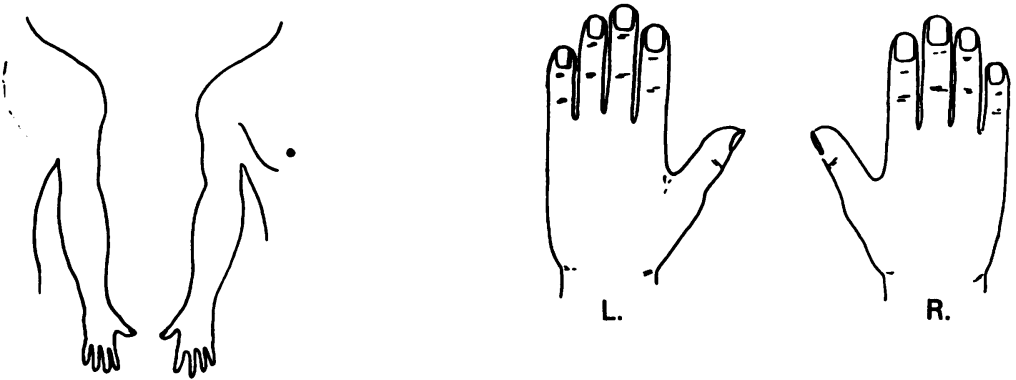

R.

\section{BACK RIGHT FRONT}

Fig. 2: Two pages from a returned questionnaire showing the typical type of response obtained $\left(\mathrm{Cl}_{1}=\right.$ an individual's commonest injury; $\mathrm{C3}_{\text {- }}$ an individual's third commonest injury; C4 = an individual's fourth commonest injury).

climbing was fairly evenly distributed among the "extreme grades". Only 17 reported no injuries at all. There was no correlation between standard of difficulty of climbing and number or type of injury. Out of the total number of injuries the majority $(89 \%)$, as expected, involved the upper limb.

21 individuals had sought professional help, but this was usually to a general practitioner $(78 \%)$. Most of these were unimpressed at the treatment received, with an "if you go climbing, what do you expect" attitude commonly encountered.

Lower limb injuries were confined to a scattering of knee ligament sprains, adductor sprains from wide "bridging" (Fig. 3) and one case of chronic patellar tendinitis, originating from an injury caused by a strenuous "rockover" (a manoeuvre where the whole body weight is transferred over a flexed knee, which you then stand up on).

The upper limb injuries are broken down by region as below.

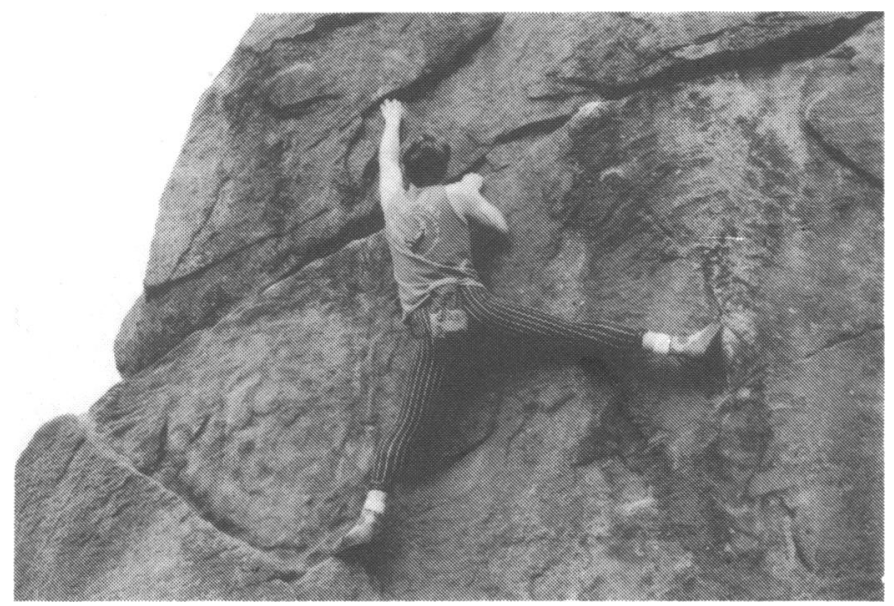

Fig. 3: Wide "bridging" can cause adductor sprains.

\section{The Shoulder}

A total of 16 shoulder injuries were reported, the majority being some variety of rotator cuff tear. Two climbers had suffered from what appeared to be an impingement syndrome. As the upper limb is used for a large amount of the time at or above shoulder height while climbing, it is perhaps surprising that this problem is not encountered more commonly.

\section{The Elbow}

There were 19 elbow injuries as in Table I. Lateral epicondylitis was far more common than medial epicondylitis but the commonest was "anterior elbow pain". This was often associated with repetitive pull-ups, or traversing on climbing walls. Climbing involves the use of the arms predominantly in the pronated and semi-flexed position. In this position the majority of the strain is taken through brachialis (I have confirmed this electromyographically). This injury is therefore presumed to be brachialis sprain or in some cases of overuse, brachialis tendinitis or "climbers elbow".

\section{The Wrist and Hand}

Hand and wrist injuries were the most commonly reported, having occurred in $50 \%$. The mechanism was predominantly one of falling while holding onto very small holds, although a significant number reported pain in finger joints and along finger flexor tendons after frequent training sessions on indoor climbing walls.

Of the hand injuries, the most commonly affected site was the proximal interphalangeal joint, or proximal phalanx of the middle and ring fingers, with pain or injury reported at this site in $69 \%$. On examining many responders, tenderness could be elicited primarily at the insertion of flexor digitorum superficialis into the base of the middle phalanx, and pain was increased by stressing this tendon.

I feel that this represents superficialis tenoperiostitis, or 
small tears at its insertion ("climbers finger"). The reason for this, is the type of hold used by over $90 \%$ of climbers on small holds (Fig. 4). This puts flexor digitorum profundus at a mechanical disadvantage, transferring the majority of the load onto flexor digitorum superficialis. It also puts the pulley system of the flexor sheath at the proximal phalanx under considerable strain and on examination a number of climbers had increased "bowstringing" of the flexor tendons following acute injury. The most vulnerable digits, as mentioned above, were the ring and middle fingers, affected in $42 \%$ and $53 \%$ of cases respectively.

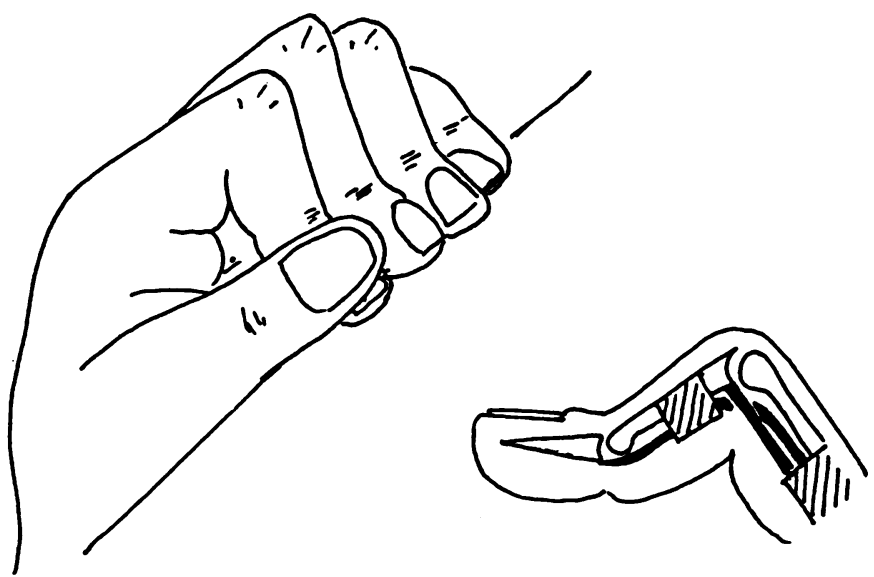

Fig. 4: A diagram of the most commonly used hold - "crimping" - where there is flexion of the proximal interphalangeal joint and extension of the digital interphalangeal joint putting flexor digitorum profundus at a mechanical disadvantage.

The first metacarpophalangeal joint was also a common site of pain. This was almost invariably associated with using "pinch grips", when a projecting hold is squeezed between the thumb and fingers, often spraining the ulnar collateral ligament.

Other reported injuries were sprains of the collateral ligaments of the proximal interphalangeal joints, a mallet finger and tenosynovitis of the wrist extensors. Wrist "sprains" were also commonly reported.

\section{DISCUSSION}

A literature search has revealed only one previous paper on climbing injuries - a collection of four case reports (Bannister and Foster, 1986). As rock climbing becomes increasingly popular and the original dangers from falling are reduced by the quality of modern protection gear, injury problems related to the difficulty of the climbing and to overuse, assume increasing importance. With the competition for sponsorship and the limited fame of appearing in the climbing magazines becoming fiercer, the temptation to push beyond physical ability or overtrain grows ever stronger.

The percentage of injuries sustained during training, reflects not only the number of climbers training during the winter months, but perhaps also the current state of ignorance within the sport about training methods. For instance, the average time spent warming up before climbing or training was eight minutes and $45 \%$ of the responders didn't warm up at all! In an attempt to reduce the incidence of training injury, the British Mountaineering
Council will shortly be producing a book - "Training for Rock Climbing", outlining suitable training schedules.

Further studies are already underway, looking in more detail at the spectrum of hand injuries, and as to whether using the fingers as weight bearing structures predisposes to premature osteoarthrosis.

This study is obviously not definitive, but has served to highlight the commoner types of problem affecting the modern climber, some of which appear to be unique to this increasingly popular sport.

\section{References}

Bannister, P. and Foster, P., 1986 "Upper Limb Injuries Associated with Rock Climbing". Brit.J.Sports Med. 20: 55.

British Mountaineering Council Annual Report 1987.

\section{APPENDIX}

Part of questionnaire relating to injuries

\section{INJURIES}

\section{Climbing}

Indicate the site of your commonest climbing injury on the diagrams by $\mathrm{C} 1$ (other than by falling!) (including muscle tears and sprains)

1) Was this injury associated with a particular move? If yes which one?

2) What was the duration and intensity (severe - moderate - annoying) of the pain?

Please repeat above for second commonest $-\mathrm{C} 2$, etc

C2 1)

2)

3 1)

C4 1)

2)

C5 1)

Which was your worst injury?

Do you treat your own injuries?

If yes what treatment do you use?

Have you sought a professional opinion?

If yes was it a Hospital Doctor

GP

Physiotherapist

Osteopath

Other

What diagnosis were you given?

What treatment did you receive and was it effective?

Do you warm up before climbing/training?

If yes how long for and what do you do?

When using small holds which hold do you use? (see diagram). Please indicate -

Most of the time Some of the time Infrequently or never

Training

Have you been injured during training?

Please indicate site of injury/ies with T1, T2, etc. on the diagram.

Please note below if any injury was associated with a particular move or exercise and the duration and intensity of the pain. Also any professional diagnosis and treatment and the outcome.

Comments and additional information 